\title{
Guianacara dacrya, a new species from the rio Branco and Essequibo River drainages of the Guiana Shield (Perciformes: Cichlidae)
}

\author{
Jessica H. Arbour ${ }^{1}$ and Hernán López-Fernández ${ }^{1,2}$
}

A new species of Guianacara is described from tributaries of the Essequibo River and the rio Branco in Guyana and northern Brazil. Guianacara dacrya, new species, can be diagnosed from all congeners by the possession of a unique infraorbital stripe and by the shape of the lateral margin of the lower pharyngeal jaw tooth plate. Guianacara dacrya can be further distinguished from G. geayi, G. owroewefi, G. sphenozona and G. stergiosi by the possession of a thin midlateral bar, from G. cuyunii by the possession of dusky branchiostegal membranes and from G. oelemariensis by the possession of two supraneurals. This species differs from most congeners by the presence of white spots on the spiny portion of the dorsal fin, the placement of the midlateral spot, the presence of filaments on the dorsal, anal and in rare cases the caudal-fin and from at least the Venezuelan species by several morphometric variables. Guianacara dacrya is known from the Essequibo, Takutu and Ireng River basins of Guyana and possibly from the rio Uraricoera in the rio Branco basin in Brazil. A key to the species is provided.

Describimos una especie nueva de Guianacara de las cuencas del río Essequibo y el río Branco en Guyana y el norte de Brasil. Guianacara dacrya, especie nueva, puede distinguirse de todos sus congéneres por la presencia de una barra infraorbital modificada y por la forma única del margen lateral de la placa dentada de la mandíbula faríngea inferior. Adicionalmente, Guianacara dacrya se distingue de G. stergiosi, G. owroewefi, G. sphenozona y G. geayi por poseer una barra medio-lateral fina, de G. cuyunii por poseer membranas branquistegales grises y de G. oelemariensis por poseer dos supraneurales. La especie nueva se distingue de la mayoría de sus congéneres por la presencia de manchas blancas en la porción espinosa de la aleta dorsal, la posición de la mancha medio-lateral, la presencia de filamentos en la aleta dorsal, anal y en algunos casos en la caudal, y de al menos las especies venezolanas por varias características morfométricas. Guianacara dacrya se conoce de las cuencas del ríos Essequibo, Takutu e Ireng en Guyana y posiblemente del río Uraricoera en la cuenca del río Branco en Brasil. Se presenta una clave de especies.

Key words: Brazil, Geophagini, Guyana, Neotropical region, South America.

\section{Introduction}

The Neotropical cichlid genus Guianacara Kullander \& Nijssen, 1989 is the sister-group to Mazarunia Kullander, 1990 with which it forms the only clade of cichlids endemic to the Guiana shield and its surrounding lowlands (López-Fernández et al., 2010). Guianacara currently consists of four species from Surinam and French Guiana (Kullander \& Nijssen, 1989; Kullander, 2003): Guianacara geayi (Pellegrin, 1902), G. oelemariensis, G. sphenozona and G. owroewefi Kullander \& Nijssen, 1989, and two species from the Venezuelan portion of the Guiana Shield: G. stergiosi and G. cuyunii LópezFernández, Taphorn \& Kullander, 2006. Guianacara specimens from the Essequibo and Potaro River basins and from rio
Branco tributaries in the Rupununi region in Guyana (former British Guiana) have historically been referred to as Aequidens (= Guianacara) geayi (Pellegrin, 1902; Lowe-McConnell, 1969) and in more recent museum records as Guianacara sphenozona or G. owroewefi (e.g., ROM, ANSP, AUM, pers. obs.). While these names correspond to similar species present in drainages in Surinam and French Guiana, our observations revealed that none of these species is actually present in Guyana.

Both distributional (e.g., Lowe-McConnell, 1969; Sabaj et $a l ., 2008$ ) and genetic (Lovejoy \& de Araújo, 2000) evidence suggests that, while some species (e.g., Cichla ocellaris, Potamorrhaphis guianensis and Rhinodoras armbrusteri) of freshwater fishes can traverse the floodplains of the Rupununi

125 Willcocks Street, Department of Ecology and Evolutionary Biology, University of Toronto, Ontario M5S 3B2, Canada. jessica.arbour@utoronto.ca

${ }^{2} 100$ Queen's Park, Department of Natural History, Royal Ontario Museum, Toronto, Ontario M5S 2C6, Canada. 
savannas in southern Guyana and move between the rio Branco (Amazon basin) and the Essequibo basin during the wet season, other species (e.g., Cichla temensis) are not able to move between the two basins. Understanding this dual pattern of distribution requires a clear taxonomic understanding of species in the area as a requisite to distinguish those that are able to move freely between the basins and those who are limited in their movements by ecological or other kinds of filters (e.g., Winemiller et al., 2008). We examined specimens of Guianacara from both the Essequibo and the Takutu-Ireng-Branco basins in Guyana and Brazil and determined that populations on either side of the Rupununi savannas are morphologically indistinguishable from each other, but are clearly diagnosable as a species different from all other described Guianacara. In this paper, we provide a description for this new Guyanese species, describe its known distribution in the Essequibo and Branco River basins, and provide an updated identification key for all named species of Guianacara.

\section{Material and Methods}

Morphological counts and measurements follow Kullander (1986) and Kullander \& Nijssen (1989). Measurements were obtained using digital calipers to the nearest $0.1 \mathrm{~mm}$. Fin ray, scale and gill raker counts were made using a dissecting microscope. Scale row nomenclature follows Kullander (1996) in which rows above the lower lateral line are named E1, E2, etc., moving dorsally and rows below the lower lateral line are named $\mathrm{H} 1, \mathrm{H} 2$, etc., moving ventrally. Counts are expressed as the observed count followed by the number of individuals examined that had that value in parenthesis, with the holotype value indicated with a*. Descriptions of the midlateral bar and the midlateral spot follow López-Fernández et al. (2006). Ontogenetic change in pigmentation patterns is described when necessary as juvenile specimens from all species generally exhibit a dark, predominantly uniform midlateral bar, but adults may have either a midlateral bar, midlateral spot or both depending upon the species being examined (Kullander \& Nijssen, 1989; López-Fernández et al., 2006). Type material included all measured (and some non-measured) specimens and encompassed the geographic distribution and range of ontogenetic and morphological variation for this species.

For statistical analyses, continuous morphological variables were corrected for the effect of body size by regression against SL (Reist, 1986); the residuals were subsequently compared, using ANOVA's and Tukey pairwise comparisons ( $\alpha=0.05$ for both), to compare the new species with previously described species of Guianacara to which it is most superficially similar (G. cuyunii and G. stergiosi). Body shape was contrasted between drainages using a relative warp analysis (using the TPS software package Rohlf, 2003) of 16 coordinate pairs (Fig. 1), including homologous landmarks and semilandmarks from curves re-sampled by length (Rohlf \& Slice, 1990; Bookstein, 1991; Bookstein, 1996; Rohlf, 2002). The coordinates were corrected for bending in the position of the body using the 'un-bend' utility in the TPS software package, using the anterior tip of the snout, dorsal insertion point of the pectoral fin, and the intersection of the lower lateral line with the end of the caudal peduncle.

Vertebral counts were made using specimens cleared and stained (c\&s) with alizarin red and alcian blue (for bone and cartilage respectively; Taylor \& van Dyke, 1985) and include the last half centrum. Museum abbreviations follow Leviton et al. (1985) and Sabaj-Pérez (2010).

\section{Results}

\section{Guianacara (Guianacara) dacrya, new species Figs. 2-6}

Holotype. UG/CSBD 1666, (ex. ANSP 177130), $79.2 \mathrm{~mm} \mathrm{SL}$, Guyana, Potaro-Siparuni (Region 8), Water Dog Falls, Burro Burro River, Essequibo Drainage, 0440'48”N 5850’46”'W, 20 Nov 1997, E. McBirney, W. Prince \& D. Jaferally.

Paratypes. Guyana, upper Takutu-upper Essequibo (Region 9): ANSP 179073, 1, $87.1 \mathrm{~mm}$ SL, Yuora River, Takutu-Branco drainage, $6.7 \mathrm{~km}$ NE of Karasabai on road to Tiger Creek village, $04^{\circ} 03^{\prime} 14^{\prime \prime} \mathrm{N}$ $59^{\circ} 29^{\prime} 07^{\prime \prime} \mathrm{W}, 31$ Oct 2002, M. H. Sabaj, J. Armbruster \& M. Thomas; ROM 85732, 13, 20.5-48.9 mm SL, Old Lady Pond, Essequibo

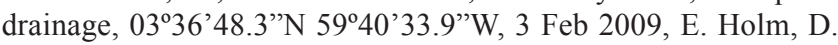
Taphorn, G. Orti, K. Sugrim, C. Sugrim, C. Bernard \& C. Lopez; ROM 85724, 1, $51.2 \mathrm{~mm}$, Rupununi River, Essequibo drainage,

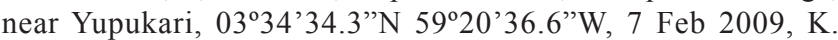
Winemiller, C. Montana, M. Tobler, G. Orti \& R. King; ROM 85888, 1, $76.9 \mathrm{~mm}$, Rupununi River, Essequibo drainage, at

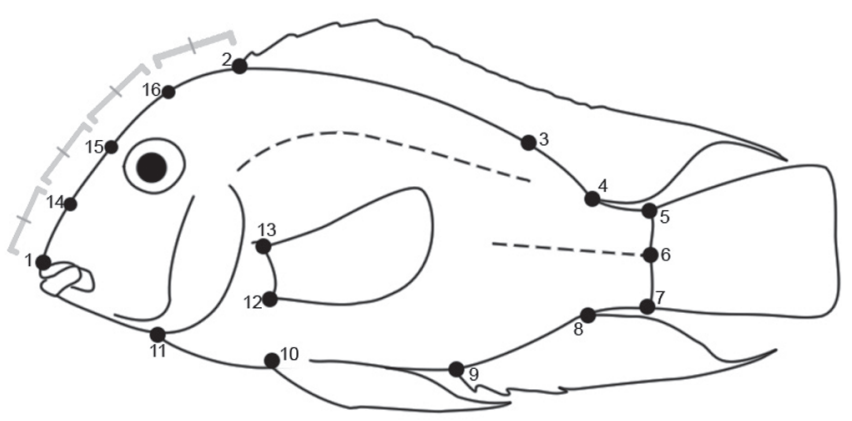

Fig. 1. Landmarks used in a geometric morphometric analysis of Guianacara dacrya: 1) anteriormost tip of the snout, 2) anterior insertion of the dorsal fin, 3 ) insertion of last dorsal spine, 4) posterior insertion of the dorsal fin, 5) dorsal insertion of the caudal fin, 6) posteriormost scale of the lower lateral line on the caudal peduncle, 7) ventral insertion of the caudal fin, 8) posterior insertion of the anal fin, 9) anterior insertion of the anal fin, 10) anterior insertion of the ventral fin, 11) caudalmost edge of the isthmus, 12) ventral insertion of the pectoral fin, 13) dorsal insertion of the pectoral fin. Semilandmarks (14-16) were estimated from curves re-sampled by length between the tip of the snout and the anterior insertion of the dorsal fin. 
Dadanawa, 0249'54”N 59³1'41.2”W, 31 Jan 2009, H. LópezFernández, C. Bernard, K. Winemiller \& D. Taphorn; ROM 85772 , 7, 20.1-95.9 mm SL, Pirara River, Takutu-Branco drainage, $40 \mathrm{~km}$ N of Lethem, 03³7'17.3'N 5940'29.4'W, 3 Feb 2009, H. LópezFernández, C. Bernard, K. Winemiller \& D. Taphorn; INPA 34980 , 1, 61.1 mm SL, Pirara River, Takutu-Branco drainage, $40 \mathrm{~km} \mathrm{~N}$ of Lethem, 03³7'17.3"N 5940’29.4"W, 3 Feb 2009, H. LópezFernández, C. Bernard, K. Winemiller \& D. Taphorn; ROM 85721, 11, 16.2-55.6 mm SL (1, 38.0 mm SL), Pirara River, Takutu-Branco drainage, 03³7'29'N 5940’38’W, 4 Feb 2009, H. López-Fernández, R. King, D. Bloom \& D. Taphorn; INPA 34979, 4, 45.5 to $55.4 \mathrm{~mm}$ SL, Pirara River, Takutu-Branco drainage, 033'ㄹ' $29^{\prime \prime N} 59^{\circ} 40^{\prime} 38^{\prime \prime} \mathrm{W}$, 4 Feb 2009, H. López-Fernández, K. Winemiller, R. King, D. Bloom \& D. Taphorn; ROM 85751, 8. 27.1-82.0 mm, Sawariwau River, Takutu-Branco drainage, at Potarinao road crossing, $03^{\circ} 02 ' 22^{\prime}$ N 5945'41.6”W, 1 Feb 2009, H. López-Fernández, C. Bernard, K. Winemiller \& D. Taphorn; ANSP 185172, 3, 34.4-85.6 mm SL, Kuyuwini River, Essequibo drainage, $60.6 \mathrm{~km}$ ENE of Kuyuwini Landing, 02 11 '35'N 58 42'15'W, 6 Nov 2003, M. H. Sabaj, J. Armbruster \& M. Hardman; ANSP 180671, 5, 31.2-89.9 mm SL, Essequibo River, $32.9 \mathrm{~km}$ SE of the mouth of Kuyuwini River, 02 00'10"N 58²7'51'W, 9 Nov 2003, M. H. Sabaj, J. Armbruster \& M. Hardman; ANSP 177129, 7, 26.3-101.7 mm SL, Yurie Creek, Essequibo River, $2.0 \mathrm{~km}$ upstream from Paddie Rock campsite, 0442'03”N 5842'44”'W, 26 Nov 1997, C. Watson, D. Torres \& M. Captain; ANSP 177133, 16, 19.5-108.5 mm SL, Essequibo River, $2.0 \mathrm{~km}$ upstream from Paddle rock campsite, $04^{\circ} 42^{\prime} 20^{\prime \prime} \mathrm{N}$ 5842'26”W, 25 Nov 1997, C. Watson, D. Torres \& E. McBirley; AUM 45171, 30, 16.3-39.9 mm SL, Takutu River, rio Branco drainage, 03³6'04'N 59³7'32'W, 29 Nov 2005, L. S. de Souza, N. Lujan, D. Taphorn, J. Hartsell, E. Liverpool \& S. Lord; AUM 38941 , 6, 31.3-95.8 mm SL (1, 95.8 mm SL), Kuyuwini River, Essequibo drainage, $19.5 \mathrm{~km} \mathrm{~W}$ from mouth of river, $02^{\circ} 14^{\prime} 28^{\prime} \mathrm{N} 58^{\circ} 30$ ' 03 ' $\mathrm{W}$, 11 Nov 2003, J. W. Armbruster, M. H. Sabaj, M. Hardman, D. Thomas, N. Lujan \& L. S. de Souza; AUM 38071, 8, 29.7-109.6 mm SL (1, 109.6 mm SL), Kuyuwini River, Essequibo drainage, $15.2 \mathrm{~km}$ E of Kuyuwini Landing, $02^{\circ} 03^{\prime} 48^{\prime \prime}{ }^{\circ} 9^{\circ} 07^{\prime} 00^{\prime \prime W}, 5$ Nov
2003, J. W. Armbruster, M. H. Sabaj, M. Hardman, D. Thomas, N. Lujan \& L. S. de Souza; AUM 36110, 2, 72.5-87.5 mm SL, Yuora River, Takutu-Branco drainage, 6.7 km NE of Karasabai, $04^{\circ} 03^{\prime} 14^{\prime \prime} \mathrm{N}$ 59²9'07'W, 31 Oct 2002, J. W. Armbruster, M. H. Sabaj, C. Allison, M. Thomas, C. Chin, D. Arjoon \& L. Atkinson; AUM 48715, 4, 66.3-116.6 mm SL (2, 100.8-116.6 mm SL), Aruwa Falls, Rupununi River, Essequibo drainage, 03³0'07'N 59²0'22”'W, 21 Nov 2007, L. S. de Souza, D. Taphorn, J. Baskin, T. Geerinckx \& J. Hwan; AUM 38827, 3, 33.9-88.5 mm SL (1, 88.5 mm SL), Kuyuwini River, Essequibo drainage, at Kuyuwini Landing, $02^{\circ} 05^{\prime} 55^{\prime} \mathrm{N}$ 59¹4'58'W, 14 Nov 2003, J. W. Armbruster, M. H. Sabaj, M. Hardman, D. Arjoon, N. Lujan \& L. S. de Souza; AUM 36111, 4, 14.9-53.5 mm SL (1, 53.5 mm SL), Rupununi River, Essequibo drainage, $5.9 \mathrm{~km}$ WSW of Sand Creek, 02 ${ }^{\circ} 57^{\prime} 60^{\prime \prime} \mathrm{N} 59^{\circ} 34^{\prime} 10^{\prime \prime} \mathrm{W}, 4$ Nov 2002, D. C. Werneke, C. Allison, M. Thomas, C. Chin \& D. Arjoon; AUM 38793, 5, 29.6-63.1 mm SL (2, 41.5-63.1 mm SL), Kuyuwini River, Essequibo drainage, $32.9 \mathrm{~km}$ SE from mouth of river, 0200'10"N 58 27'51'W, 9 Nov 2003, J. W. Armbruster, M. H. Sabaj, M. Hardman, D. Arjoon, N. Lujan \& L. S. de Souza; AUM 38124, 3, 29.1-45.0 mm SL (1, 45.0 mm SL), Kuyuwini River, Essequibo drainage, $02^{\circ} 02^{\prime} 51^{\prime \prime N} 59^{\circ} 00^{\prime} 09^{\prime}$ W, 5 Nov 2003, J. W. Armbruster, M. H. Sabaj, M. Hardman, D. Arjoon, N. Lujan \& L. S. de Souza; AUM 48431, 1, 66.1 mm SL, Rupununi River, Essequibo drainage, 0339'29"N 59²1'38'W, 9 Nov 2007, L. S. de Souza, D. Taphorn, J. Baskin, T. Geerinckx \& J. Hwan; AUM 36190, 7, 14.152.9 mm SL (5, 43.5-52.9 mm SL), Pirara, Takutu-Branco drainage, 0338'55'N 5941'20"W, 2 Nov 2002, J. W. Armbruster, M. H. Sabaj, M. Thomas \& D. Arjoon; AUM 36112, 1, 94.9 mm SL (1, $94.9 \mathrm{~mm} \mathrm{SL}$ ), Takutu River, Branco drainage, $2.75 \mathrm{~km}$ W of Saint Ignatius, $03^{\circ} 21^{\prime} 18^{\prime}$ 'N 5949'51'W, 5 Nov 2002, J. W. Armbruster, M. H. Sabaj, D. Werneke, C. Allison, M. Thomas, C. Chin \& D. Arjoon; AUM 48137, 8, 34.8-75.6 mm SL, (8, 34.8-75.6 mm SL), Pirara River, Takutu-Branco drainage, 0337'31'N 5940'34”W, 19 Nov 2007, L. S. de Souza, D. Taphorn, J. Baskin, T. Geerinckx \& J. Hwan; AUM 44709, 3, 21.9-99.2 mm SL (2, 45.3-99.2 mm SL), Takutu River, Branco drainage, near Lethem, $03^{\circ} 28^{\prime} 14^{\prime \prime} \mathrm{N}$ 5948'36”'W, 27 Nov 2005, L. S. de Souza, N. Lujan, D. Taphorn, J.

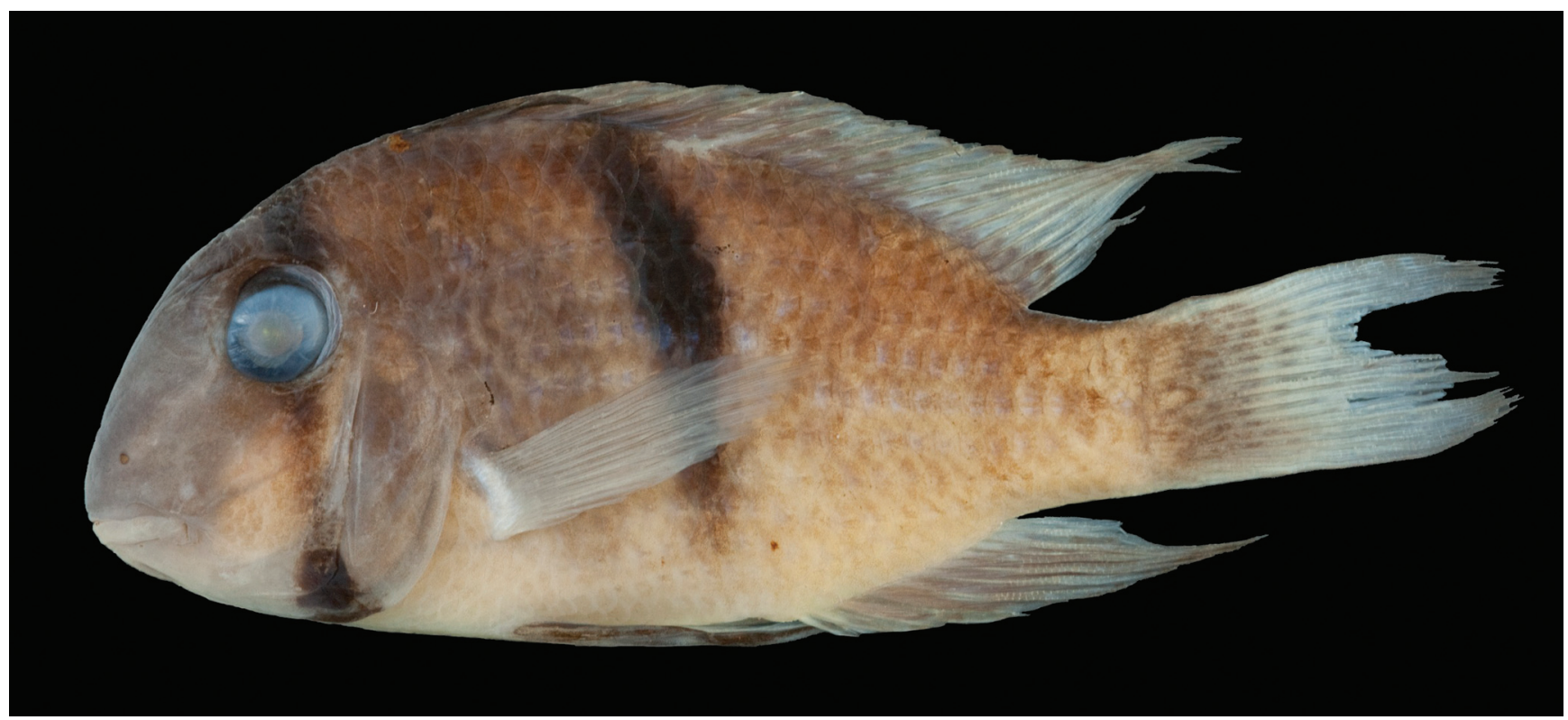

Fig. 2. Guianacara dacrya, holotype, UG/CSBD 1666, 79.2 mm SL, Guyana, Potaro-Siparuni (Region 8), Water Dog Falls, Burro Burro River, Essequibo River drainage. 


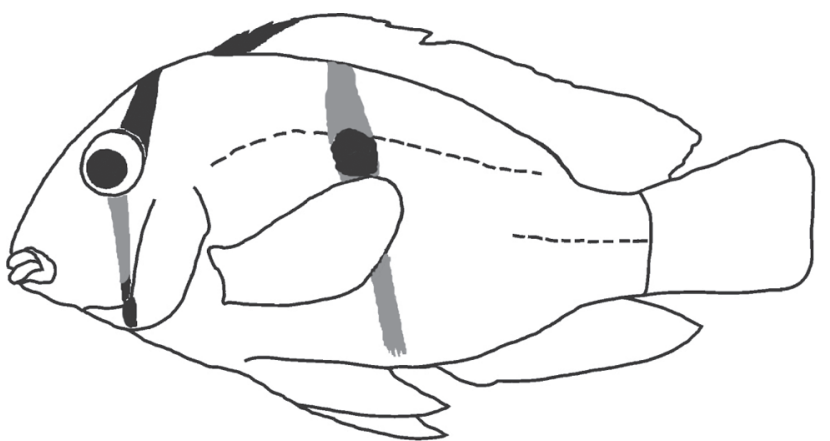

a Guianacara dacrya

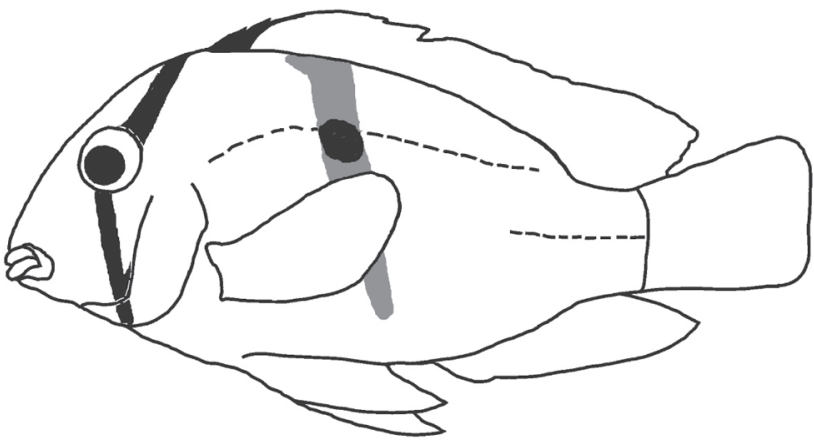

C Guianacara stergiosi

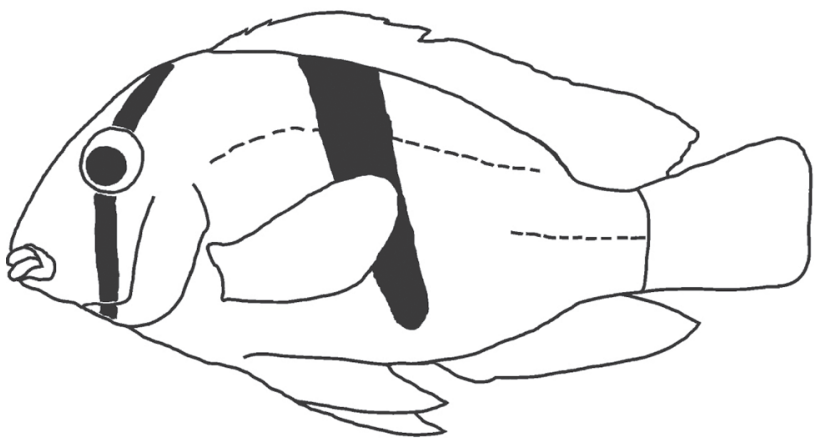

e Guianacara geayi

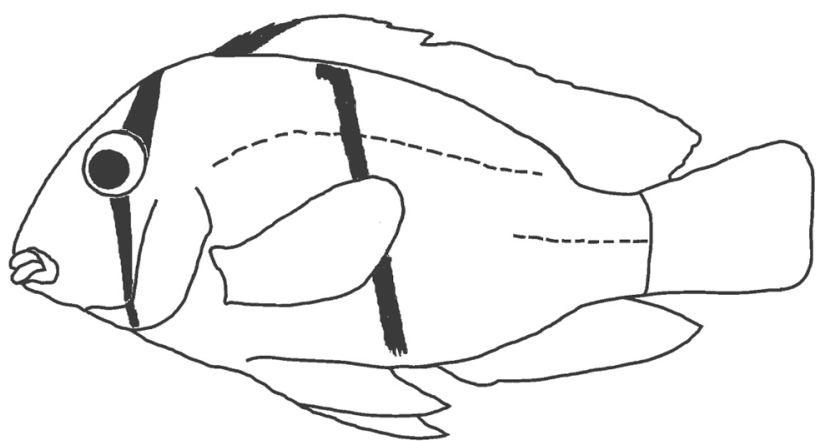

b Guianacara cuyunii

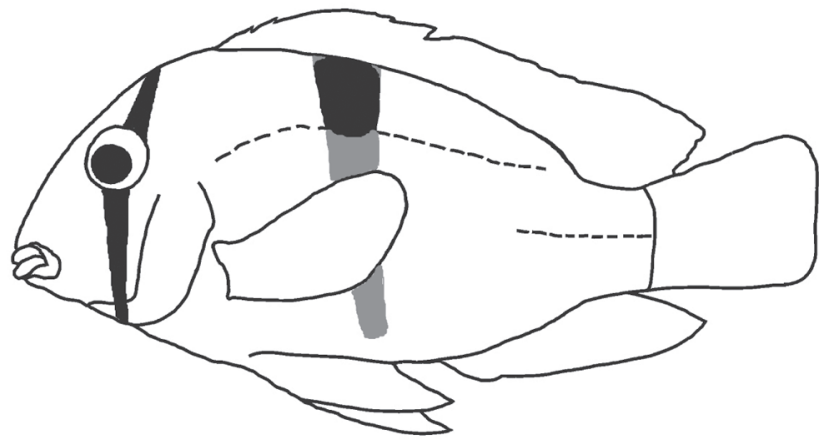

d Guianacara sphenozona

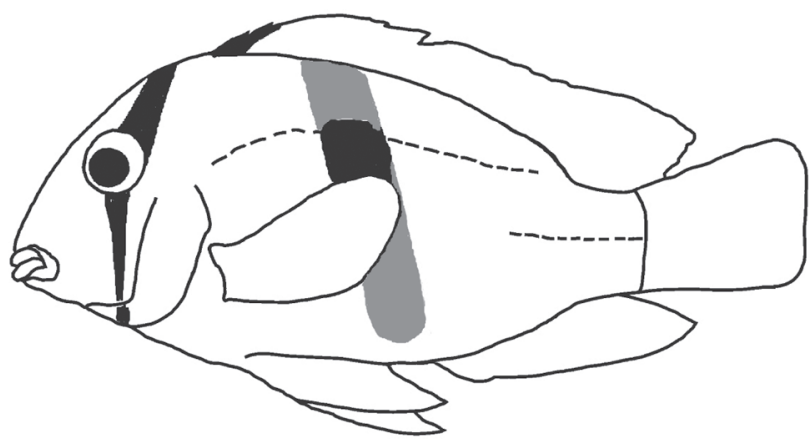

f Guianacara owroewefi

Fig. 3. Colour patterns distinguishing species of Guianacara in the subgenus Guianacara.

Hartsell, E. Liverpool \& S. Lord; AUM 48812, 2, 42.1-88.3 mm SL, (2, 42.1-88.3 mm SL) Rupununi River, Essequibo Drainage, at Massara Landing, 0353'42'N 59¹7'37'W, 22 Nov 2007, L. S. de Souza, D. Taphorn, J. Baskin, T. Geerinckx \& J. Hwan; AUM 44521, 4, 12.5-102.5 mm SL (1, 66.7 mm SL), Bununi Creek, Rupununi River, Essequibo drainage, $15.8 \mathrm{~km}$ W of Massara, 0352'10”N 59²6'02'W, 25 Nov 2005, L. S. de Souza, N. Lujan, D. Taphorn, J. Hartsell, E. Liverpool \& S. Lord; AUM 48336, 4, 62.9-119.8 mm SL (2, 62.9-90.8 mm SL), Rupununi River, Essequibo drainage, at Dadanawa Ranch, 0249'52"N 59³1'40”W, 14 Nov 2007, L. S. de Souza, D. Taphorn, J. Baskin, T. Geerinckx \& J. Hwan; AUM 38155, 2, 46.6-106.6 mm SL, (1, 106.6 mm), Kuyuwini River, Essequibo drainage, $60.6 \mathrm{~km}$ ENE of Kuyuwini Landing, $02^{\circ} 11^{\prime} 35^{\prime \prime N} 58^{\circ} 42^{\prime} 15^{\prime \prime}$ W, 6 Nov 2003, J. W. Armbruster, M. H. Sabaj, M. Hardman, D. Arjoon, N. Lujan \& L. S. de Souza; AUM 37960, 4, 50.0-69.1 mm SL (2, 50.0-69.1 mm SL), Kuyuwini Landing,
$1.14 \mathrm{~km}$ upstream from mouth of river, $02^{\circ} 16^{\prime} 02^{\prime \prime} \mathrm{N} 58^{\circ} 20^{\prime} 15^{\prime \prime} \mathrm{W}, 7$ Nov 2003, J. W. Armbruster, M. H.Sabaj, M. Hardman, D. Arjoon, N. Lujan \& L. S. de Souza; AUM 39046, 4, 46.3-80.0 mm SL (3, 55.4-80.0 mm SL), Yukanopito Falls, Kuyuwini River, Essequibo drainage, $01^{\circ} 54$ '53"N 58 31'14"W, 9 Nov 2003, J. W. Armbruster, M. H. Sabaj, M. Hardman, D. Arjoon, N. Lujan \& L. S. de Souza; AUM 38191, 2, 30.5-80.4 mm SL (1, 80.4 mm SL), Kuyuwini River, Essequibo drainage, $48.4 \mathrm{~km}$ Kuyuwini Landing, $02^{\circ} 03$ ' 54 '” 5848'33"W, 9 Nov 2002, J. W. Armbruster, M. H. Sabaj, M. Hardman, D. Arjoon, N. Lujan \& L. S. de Souza, Guyana, PotaroSiparuni (Region 8): ANSP 177136, 5, 32.3-65.7 mm SL, Red Hill Creek, Siparuni River, Essequibo drainage, between Levi Falls and Blackwater camp, 0444'14'N 5859'17'W, 5 Dec 1997, G. Watkins et al.; ANSP 177135, 21, 17.4-81.1 mm SL, Levi Fall, Siparuni River, Essequibo drainage, 0444'41'N 5900'18'W, 4 Dec 1997, G. Watkins et al.; ANSP 177134, 6, 21.4-80.7 mm SL, Tumble 
Down Creek, Siparuni River, Essequibo drainage, 04'48'39'N 5851'11"W, 8 Dec 1997, G. Watkins et al.; ANSP 177130, 1, 79.2 mm SL, Water Dog Falls, Burro Burro River, Essequibo drainage, 0440'48”N 5850'46”'W, 20 Nov 1997, E. McBirney, W. Prince \& D. Jaferally; AUM 50647, 1, $42.1 \mathrm{~mm}$ SL, Essequibo River, at Pisham-Pisham Rapids, 04²5'54"N 58²9'11'W, 4 Dec 2005, L. S. de Souza, N. Lujan, D. Taphorn, J. Hartsell, E. Liverpool \& S. Lord; AUM 28122, 2, 37.7-44.2 mm SL, Potaro River, Essequibo drainage, $05^{\circ} 18$ '14”N 59¹8'40”W, 25 Oct 1998, L. M. Page, J. Armbruster, M. H. Sabaj, M. Hardman, J. Knouft \& W. Prince; AUM 45333, 2, 20.6-44.2 mm SL (1, $44.2 \mathrm{~mm}$ SL), Essequibo River, 5 Dec 2005, L. S. de Souza, N. Lujan, D. Taphorn, J. Hatsell, E. Liverpool \& S. Lord.

Non-type material. Guyana, upper Takutu-upper Essequibo (Region 9): ANSP 179494, 1, 67.3 mm SL, Takutu River, Branco drainage, $2.75 \mathrm{~km}$ W of Saint Ignatius, 03²1'18'N 5949'51' 'W, 5 Nov 2002, M. H. Sabaj, J. Armbruster \& M. Thomas; ANSP 179495, 6, 10.5-39.1 mm SL, Pirara River, Takutu-Branco drainage, 3.5 km NNW of Pirara, 0338'55'N 5941'20”W, 2 Nov 2002, M. H. Sabaj, J. Armbruster \& M. Thomas; AUM 46490, 1, $45.5 \mathrm{~mm}$ SL, Manari Creek, Takutu River, rio Branco drainage, 0327'04”N 5946'24'W, 28 Nov 2005, L. S. de Souza, N. Lujan, D. Taphorn, J. Hartsell, E. Liverpool \& S. Lord; AUM 44671, 13, 13.1-51.9 mm SL, Pirara River, Takutu-Branco drainage, at Pirara Ranch, 0337'31'N 5940'37'W, 26 Nov 2005, L. S. de Souza, N. Lujan, D. Taphorn, J. Hartsell, E. Liverpool \& S. Lord; AUM 44670, 1, $70.1 \mathrm{~mm}$ SL, Pirara River, Takutu-Branco drainage, Pirara Ranch, 0337'31'N 5940'37'W, 26 Nov 2005, L. S. de Souza, N. Lujan, D. Taphorn, J. Hartsell, E. Liverpool \& S. Lord; ANSP 177128, 6, 25.2-103.2 mm SL (2 c\&s), Essequibo River, 0444'22"N 5842'23'"W, 24 Nov 1997, C. Watson, D. Torres \& D. Siegel; ANSP 185173, 9, 24.4-80.7 mm SL, Kuyuwini River, Essequibo drainage, $15.2 \mathrm{~km}$ E of Kuyuwini Landing, 02 03 '48”N 5907'00”'W, 5 Nov 2003, M. H. Sabaj, J. Armbruster \& M. Hardman; ANSP 185171, 2, 61.4-73.3 mm SL, Kuyuwini River, Essequibo drainage, 48.4 km E of Kuyuwini Landing, 0203'54"N 5848'33”W, 6 Nov 2003, M. H. Sabaj, J. Armbruster \& M. Hardman; ANSP 185177, 1, 60.8 mm SL, Madkauwau Creek, Kuyuwini River, Essequibo drainage, between Kuyuwini Landing and Parabara village, $02^{\circ} 05^{\prime} 48^{\prime} \mathrm{N}$ 59¹4'36"W, 4 Nov 2003, M. H. Sabaj, J. Armbruster \& M.

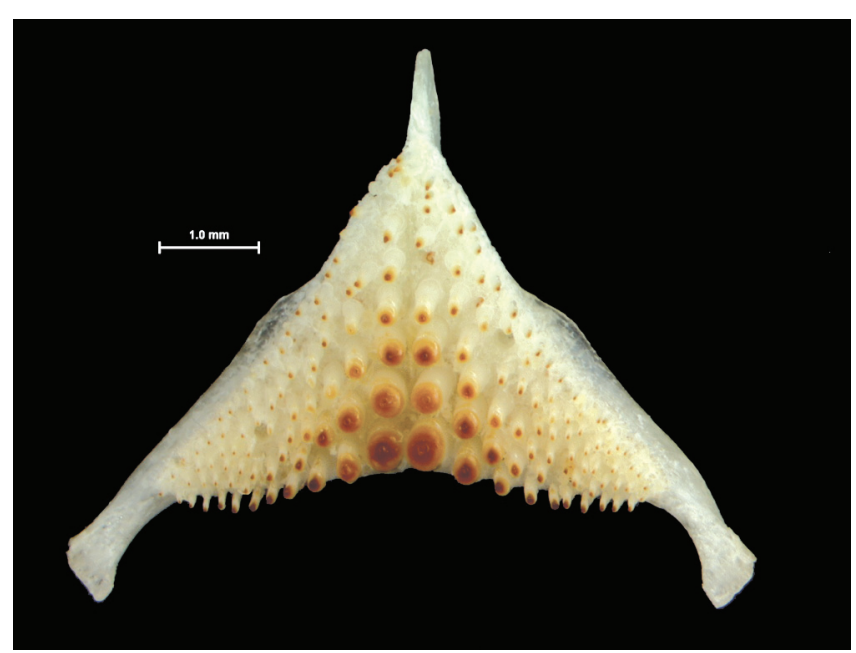

Fig. 4. Guianacara dacrya, ROM 85772, 60.9 mm SL. Lower pharyngeal jaw in occlusal view.
Hardman; ANSP 185176, 4, 32.4-68.4 mm SL, Kuyuwini River, Essequibo drainage, $1.14 \mathrm{~km}$ upstream from confluence with Essequibo River, 02 ${ }^{\circ} 16^{\prime} 02$ 'N $58^{\circ} 20^{\prime} 15^{\prime}$ 'W, 7 Nov 2003, M. H. Sabaj, J. Armbruster \& N. Lujan; ANSP 180746, 4, 11.2-55.3 mm SL, Araquai Creek, Rupununi River, Essequibo drainage, $77.3 \mathrm{~km}$ SSE of Lethem, $02^{\circ} 45^{\prime} 45^{\prime \prime} \mathrm{N} 59^{\circ} 27^{\prime} 60^{\prime}$ 'W, 15 Nov 2003, M. H. Sabaj, J. Armbruster \& M. Hardman; AUM 47856, 1, 79.6 mm SL, Rupununi River, Essequibo drainage, at Kwatamang Landing, 035'05'N 5906'02”W, 5 Nov 2007, L. S. de Souza, D. Taphorn, J. Baskin, T. Geerinckx \& J. Hwan; AUM 38096, 1, 44.6 mm SL, Madkauwau Creek, Kuyuwini River, Essequibo drainage, $02^{\circ} 05^{\prime} 45^{\prime \prime N} 59^{\circ} 14$ '28"W, 4 Nov 2003, J. W. Armbruster, M. H. Sabaj, M. Hardman, N. Lujan \& L. S. de Souza; AUM 38978, 4, 14.8-40.5 mm SL, Araquai Creek, Rupununi River, Essequibo drainage, $02^{\circ} 45^{\prime} 45^{\prime}$ 'N 59 $27^{\prime} 60^{\prime \prime}$ W, 15 Nov 2003, M. H. Sabaj, M. Hardman, N. Lujan \& L. S. de Souza; Guyana, Potaro-Siparuni (Region 8): ROM 61915, 1, 15.9 mm, Essequibo River, channel at south end of Cowhead Island, 0441'57'N 5841'30'W, 8 Oct 1990, E. Holm, A. Andries, \& D. Desrochers, C. Hestick \& L. Hately.

Diagnosis. Guianacara dacrya (Figs. 2 and 3a) belongs to the subgenus Guianacara (Fig. 3) and therefore can be distinguished from G. oelemariensis (subgenus Oelemaria) by the possession of two supraneural bones, produced dorsalfin lappets and a midlateral bar. Guianacara dacrya can be distinguished from all other species in the subgenus Guianacara by the possession of an infraorbital stripe that progressively fades across the cheek (from just beneath the eye to the dorsal edge of the pre-opercle) in individuals $>45$ $\mathrm{mm}$ SL, but remains black or dark brown across the preopercle and interopercle (Figs. 2 and 3a). In all other species, the infraorbital stripe is continuous throughout ontogeny. The posterior half of the lower pharyngeal jaw toothplate of $G$. dacrya possesses a dorsally edentulous laminar expansion of the lateral margin that is not present in other species of Guianacara. Guianacara dacrya can be distinguished from G. stergiosi (Fig. 3c), G. sphenozona (Fig. 3d), G. owroewefi (Fig. 3f) and G. geayi (Fig. 3e) by the possession of a thin midlateral bar which covers three scales at its widest point, thinning to 1.5 to 2 scales, vs. never less than 3 scales. Additionally, adult G. dacrya retain the juvenile dark coloration

Table 1. Morphometric data for Guianacara dacrya. $\mathrm{SD}=$ Standard deviation.

\begin{tabular}{|c|c|c|c|c|c|}
\hline Variable & Holotype & $\mathrm{N}$ & Range & Mean & SD \\
\hline Standard length (mm) & 87.12 & 52 & $34.8-119.8$ & 62.8 & 22.6 \\
\hline \multicolumn{6}{|c|}{ Percents of Standard length } \\
\hline Body depth & 47.4 & 51 & $37.5-50.5$ & 44.5 & 3.0 \\
\hline Head length & 31.9 & 51 & $29.1-34.3$ & 32.2 & 1.3 \\
\hline Caudal-peduncle depth & 16.3 & 51 & $14.3-17.8$ & 15.7 & 0.8 \\
\hline Caudal-peduncle length & 13.8 & 50 & $12.0-16.5$ & 14.0 & 0.9 \\
\hline Pectoral-fin length & 28.0 & 51 & $25.6-35.6$ & 30.5 & 1.7 \\
\hline Pelvic-fin length & 38.0 & 50 & $28.7-44.4$ & 35.8 & 3.0 \\
\hline Last dorsal-spine length & 15.0 & 50 & $13.1-19.3$ & 16.0 & 1.4 \\
\hline \multicolumn{6}{|c|}{ Percents of Head length } \\
\hline Head width & 58.2 & 51 & $45.7-60.4$ & 53.0 & 3.5 \\
\hline Snout length & 48.2 & 51 & $25.9-56.4$ & 41.0 & 6.9 \\
\hline Orbital diameter & 35.0 & 51 & $29.5-48.5$ & 39.4 & 4.2 \\
\hline Interorbital width & 35.9 & 51 & $22.5-39.8$ & 30.2 & 5.1 \\
\hline Preorbital depth & 44.4 & 51 & $20.4-51.0$ & 34.5 & 8.6 \\
\hline
\end{tabular}




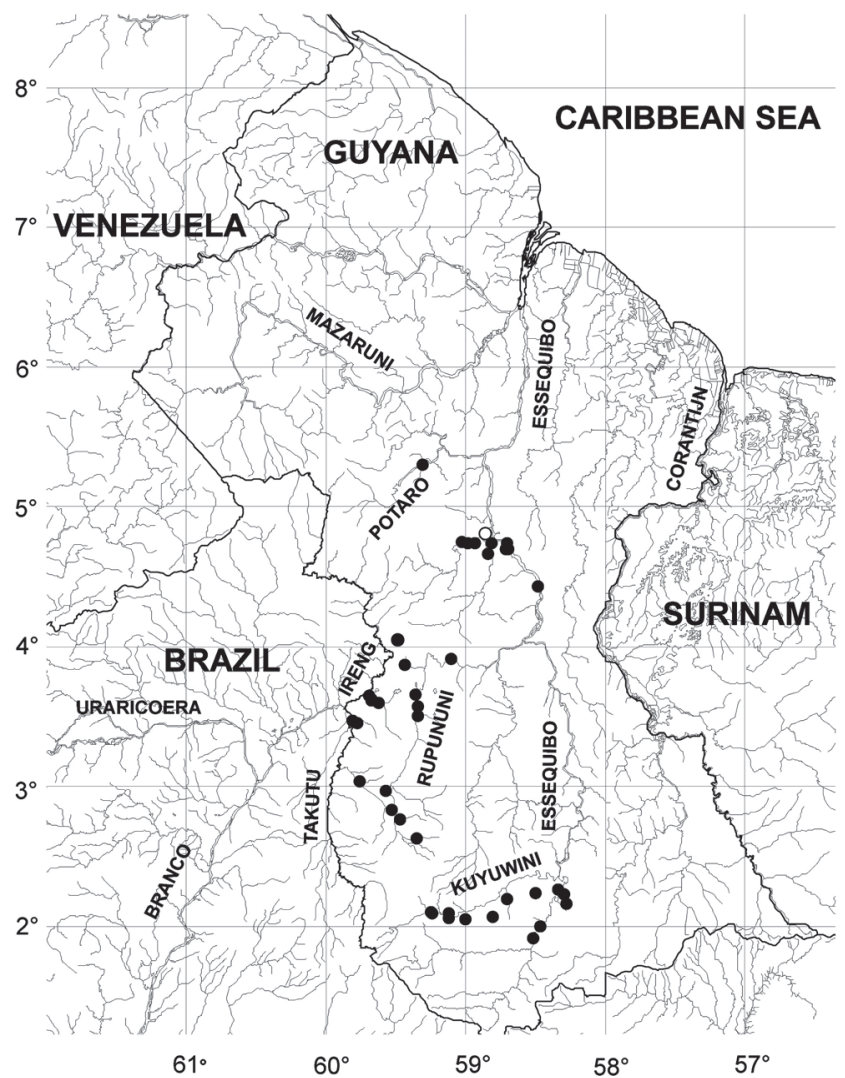

Fig. 5. Distribution of Guianacara dacrya in the Essequibo River basin in central and southern Guyana, and the rio Branco basin in south-western Guyana and north-eastern Brazil. Possible collections from the Uraricoera River drainage in Brazil are not included pending collections of adult material that would allow positive identification. Type locality indicated with an empty symbol. One symbol may represent more than one collection locality.

of the 3 anterior dorsal-fin spines and lappets, which is lost in adults of G. geayi and G. sphenozona. Guianacara dacrya can be further distinguished from $G$. owroewefi and $G$. sphenozona by the possession of white spots on both the soft and spiny portions of the dorsal-fin. When a midlateral spot is distinguishable in G. dacrya it is small, round and placed on and below the upper lateral line, similar to $G$. stergiosi. In other species the midlateral spot is large and oval shaped (G. owroewefi) or placed on and above the upper lateral line (G. sphenozona). Guianacara dacrya can be distinguished from G. stergiosi and G. cuyunii by a larger eye, a shorter and narrower head, shorter pectoral fin and by produced filaments of the anal, dorsal and (occasionally) caudal fins. Adult Guianacara dacrya can be further distinguished from adult $G$. cuyunii by a distinct midlateral spot, the possession of dusky branchiostegal membranes and a shallower body, and from adult G. stergiosi by a shorter preorbital region and longer pelvic fins.
Description. Measurements are summarized in Table 1. No apparent sexual dimorphism. Head slightly broader ventrally than dorsally. Lateral profile more convex dorsally than ventrally. Interorbital area flat to slightly convex. Dorsal head profile ascending, convex to insertion of dorsal fin; dorsal-fin base descending, convex; dorsal caudal peduncle straight or slightly concave to caudal-fin base. Ventral head profile descending, straight from lower lip to isthmus, then straight or slightly convex to pelvic-fin insertion; slightly concave to anal-fin insertion; anal-fin base ascending, slightly convex; ventral caudal peduncle straight to caudal fin base. Lips moderately wide. Lower lip fold discontinuous at dentary symphysis. Maxilla reaching to one-third distance between nostril and orbit, ascending premaxillary process nearly reaching anterior border of orbit. Opercle, preopercle, cleithrum, supracleithrum and post-temporal smooth.

E1 25(16), 26*(32); scales between upper lateral line and dorsal-fin 4(7), $41 / 2 *(40)$ anteriorly and $11 / 2$ (49) posteriorly. Scales on upper lateral line 16(2), 17*(15), 18(30), 19(2) and lower lateral line 9(15), 10*(24), 11(9). Circumpeduncular scale rows 7 above lower lateral line, 7 below, ctenoid. Scale rows between lateral lines 2 . Opercle, subopercle and cheek fully scaled, cycloid or slightly ctenoid; interopercle bare. Occipital and flank scales ctenoid. Single column of postorbital scales, cycloid. Lateral chest scales ctenoid, smaller than flank scales. Dorsal, anal, pectoral and pelvic fins naked; caudal fin with single rows of cycloid scales between rays to one quarter or one third of fin length. Accessory caudal fin extensions of lateral line dorsally between rays D3 and D4 and ventrally between rays $\mathrm{V} 4$ and $\mathrm{V} 5$.

Dorsal fin XV,9(16), XV,10*(34); anal III,7(11), III, $8 *(39)$. Dorsal spines increasing in length from first to $4^{\text {th }}-5^{\text {th }}$, decreasing to $8^{\text {th }}-10^{\text {th }}$, then relatively straight; membranous lappets behind anterior 11-13 spines, pointed, about one fifth of spine length anteriorly, decreasing posteriorly. Soft portion slightly expanded, pointed, rays 3 and 4 longest, produced into filaments in large individuals, reaching one third of caudalfin length in small individuals, reaching past the caudal fin in large individuals with filaments. Anal fin pointed, rays 3 and 4 longest, produced into filaments in large individuals, reaching one quarter of caudal fin length in small individuals to past caudal fin in large individuals with produced filaments. Caudal fin emarginate, symmetrical; in a few large, very well preserved specimens ( 96.4 to $106.6 \mathrm{~mm}$ SL, particularly ANSP 177133) first and second most dorsal caudal-fin rays produced into filaments. Pectoral fin straight along dorsal margin, rounded along caudal and ventral margins; longest at fifth ray, generally not reaching anal-fin insertion, though nearly reaching in a few specimens. Pelvic fin triangular in shape, first soft ray longest, reaching second anal spine to fourth anal ray, sometimes produced into filament.

Hemiseries in outer row of premaxilla with 10 to 13 thin, sharp, slightly recurved, unicuspid teeth, progressively smaller distally from symphysis. Three or four irregular inner rows of smaller, unicuspid teeth, separated from outermost row by a gap. Dentary hemiseries with 19 to 23 teeth in outer 


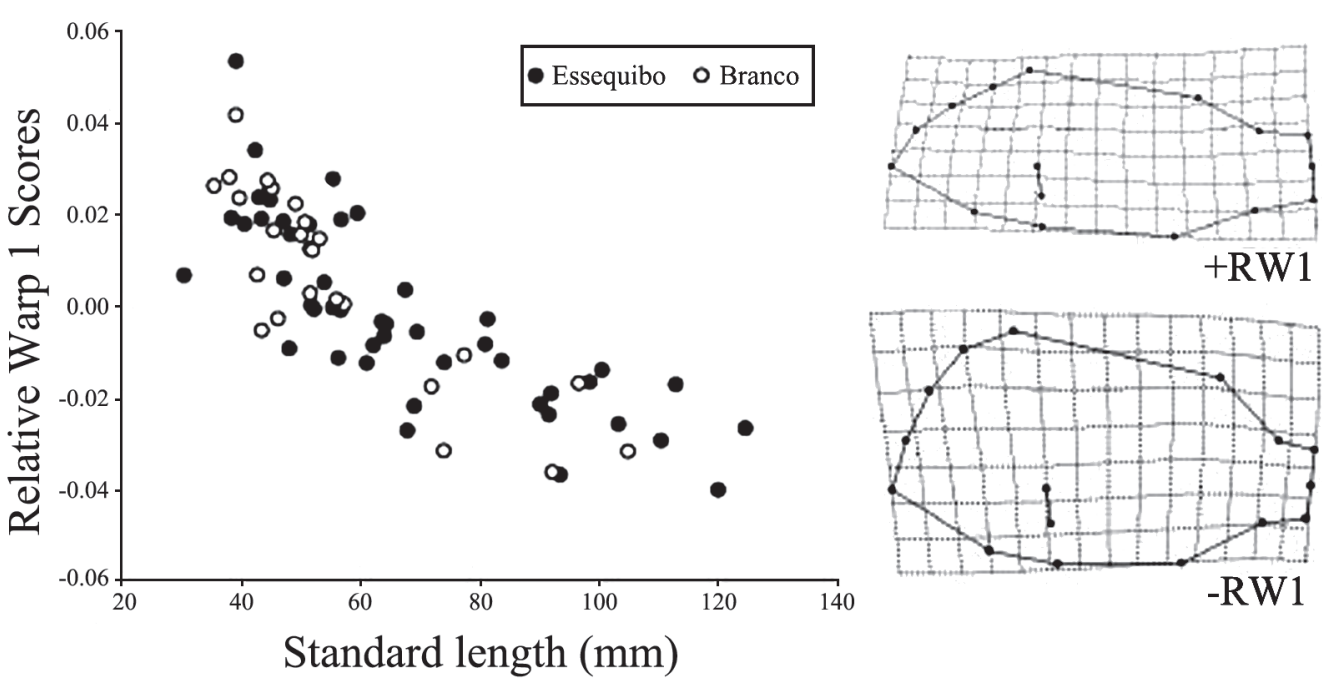

Fig. 6. Geometric morphometrics of Guianacara dacrya from the rio Branco (Takutu and Ireng River drainages in Guyana) and Essequibo River basins. Left: the relationship between body shape (RW1) and body size (SL) indicates low variation in allometry between drainages. Right: A graphical representations of the observed extremes of body shape; notice the increase in body and head depth with body size.

row, four inner rows with smaller teeth separated from outermost row by a slight gap. Dentary hemiseries separated by a small symphyseal gap, particularly in two outermost rows.

External rakers on first gill arch: 7(25), $8 *(22), 9(2)$. Gills with narrow skin cover. Lower pharyngeal tooth plate wide, length of bone $66 \%$ of width; dentigerous area $74 \%$ of width, $75 \%$ of length; 22 teeth in posterior row; 8 or 9 in median row. Lateral outer teeth unicuspid, cylindrical with slightly recurved tips, becoming progressively smaller caudally; posteromedial teeth much larger, molariform, unicuspid, with medial cusps slightly curved anteriorly. Posterior half of lateral margin of toothplate with abrupt, wing-like, dorsally edentulous laminar expansion (Fig. 4). Four tooth plates on fourth ceratobranchial, 4, 7, 7 and 3 teeth respectively from anterior to posterior plates. Vertebrae: $13+14=27(4)$.

Color in alcohol. Base colour yellowish gray to brownish yellow; snout, nape and upper lip of base colour, opercle and subopercle darker in colour. Lower lip, interopercle, lower region of preopercle, chest and lower 2 or 3 flank scale rows from chest to anal or caudal fin base whitish yellow. Branchiostegal membrane same colour as chest in small specimens, becoming progressively duskier in individuals above $40.0 \mathrm{~mm}$ SL. Flank scales darker along caudal margin. Spots slightly darker than base colour sometimes present along flank just above lower lateral line, 2-3 posterior to midlateral bar and 1 anterior to midlateral bar; spot anterior to the midlateral bar frequently expanded dorsally to base of dorsal-fin.

Supraorbital stripe on posterior third of orbit, black to dark brown, 2 to 2.5 scales wide, slightly thinner dorsally, slightly caudally directed, 3 to 3.5 scales anterior to dorsal-fin insertion. Infraorbital stripe just posterior to middle of orbit, thinner than supraorbital stripe, descending to caudal edge of interopercle. Infraorbital stripe colour lighter between the orbit and preopercle in specimens $>45 \mathrm{~mm}$ SL, becoming progressively fainter and disappearing in larger specimens; always dark on the preopercle and interopercle, sometimes becoming wider and rounder towards the ventral margin of the interopercle, forming a tear-drop shaped mark. Midlateral bar black to dark brown, frequently of uniform intensity, extending between the base of dorsal spines 7-9; the bar is three scales wide at its widest coinciding with upper lateral line, 2.5 to 3 scales wide above upper lateral line to dorsal-fin base, progressively thinning to 1.5 to 2 scales wide below upper lateral line, disappearing ventrally between $\mathrm{H} 3$ and $\mathrm{H} 4$. Midlateral bar most intense on and below upper lateral line, sometimes forming a midlateral spot 3 scales wide and 2 to 3 scales deep, extending from just above upper lateral line to just above pectoral-fin. Midlateral bar is maintained, even in largest adults $(>100 \mathrm{~mm} \mathrm{SL})$ and even if a midlateral spot is evident. Midlateral bar bounded anteriorly and posteriorly by two lighter coloured bars two to three scales in width.

Dorsal-fin dusky with three most anterior spines and associated membranes black in juveniles and most adults, but varying with preservation. Whitish spots on membranes of the soft portion and caudal half of spiny portion of dorsalfin, with white spots either of equal or smaller size than the darker, brownish or gray markings separating them. Whitish dorsal-fin spots arranged in 4 roughly parallel rows. Caudalfin dusky, with small whitish spots on membranes, not forming an obvious pattern. Anal-fin slightly dusky, whitish spots between last 2 or 3 fin rays. Pelvic-fin hyaline or slightly dusky, spine and first ray darker, gray or black. Pectoral-fin hyaline. 
Etymology. From the Greek dakryo, to shed tears or weep. In reference to the tear-streaked appearance of the infraorbital stripe. To be regarded as an adjective in feminine form.

Geographic distribution. Guianacara dacrya is known from south-western and central Guyana, as far north as the Potaro River and as far south as the Yukanopita Falls in the Essequibo River and potentially in northern Brazil as far west as the rio Uraricoera (Fig. 5, but see comments). This species is abundant in the Essequibo basin, including the drainages of its main tributaries the Rupununi, Siparuni and Kuyuwini, as well as the Takutu and Ireng drainages in the Branco River basin. Sampling has not been undertaken in the Rewa River, or its tributaries, however it is likely that further field exploration will reveal the presence of $G$. dacrya in this basin.

Habitat. As all other known species in the genus, Guianacara dacrya is generally found associated with clear water streams with moderate current, but also in seasonally flooded lagoons with no current in the Rupununi savannas. Substrate is usually sandy with nearby large rocks; large numbers of juveniles were observed in river sections formed by mixtures of pebbles and small rocks over sandy bottom, frequently with some leaf litter. Water parameters of localities where G. dacrya was collected showed a temperature range of $24.8-28.5^{\circ} \mathrm{C}$, with relatively high dissolved oxygen levels $(5.3-6.56 \mathrm{mg} / \mathrm{L})$ and very low conductivity $(27.1-34.5 \mu \mathrm{S})$ at localities in the Rupununi and Pirara (Ireng) drainages.

\section{Key to the species of Guianacara}

1a. Two supraneurals; produced lappets on tips of anterior dorsal-fin spines; dark midlateral bar present, or a faint midlateral bar with a darker midlateral spot .(subgenus Guianacara) 2

1b. One supraneural; no produced lappets on tips of anterior dorsal-fin spines; midlateral bar absent, midlateral spot present. Guianacara oelemariensis (subgenus Oelemaria)

2a. Midlateral bar thin, up to three scales wide at its widest point (two and a half to three scales at its widest point, always narrowing to one and a half or two scales at its thinnest point) ......................................... 3

2b. Midlateral bar broad, at least three scales wide at its thinnest point (four scales at its widest point, sometimes narrowing to three scales) .................................4

3a. Branchiostegal membranes whitish; infraorbital stripe continuously dark across the cheek to the interopercle in adults and juveniles................................ cuyunii

3b. Branchiostegal membranes dusky; infraorbital stripe continuously dark across the cheek to the interopercle in juveniles, but faded from orbit to dorsal edge of preopercle in adults, forming a "teardrop" mark across the preopercle and interopercle...................................G. dacrya

4a. Black pigmentation of anterior three dorsal-fin spines not retained in adults. 4b. Black pigmentation of anterior three dorsal-fin spines retained in adults...........................................

5a. Midlateral bar uniform; white spots on dorsal-fin larger than darker markings surrounding them ............... geayi 5b. Midlateral bar not uniform, midlateral spot located above the upper lateral line; white spots on dorsal-fin smaller than darker markings surrounding them....... G. sphenozona 6a. Midlateral spot small, round; midlateral bar wedge shaped (thinner ventrally than dorsally) ................... stergiosi 6b. Midlateral spot large, oval shaped; midlateral bar not wedge shape (uniform thickness) ............... . owroewefi

\section{Discussion}

We describe a new species of Guianacara, elevating the number of species in the genus to seven, and within the subgenus Guianacara to six. Guianacara dacrya appears to be the largest bodied species of Guianacara, with specimens frequently surpassing $100 \mathrm{~mm} \mathrm{SL}$, and reaching $119.8 \mathrm{~mm} \mathrm{SL}$ in the largest specimen we examined (AUM 48336). Guianacara dacrya differs from other species of Guianacara in color pattern and other morphological features (see Diagnosis). The new species is the only one with a midlateral bar as narrow as G. cuyunii. Guianacara dacrya differs from both G. cuyunii and G. stergiosi by a significantly larger eye, a shorter, and narrower head, shorter pectoral fin and the possession of produced fin filaments, which are absent from either G. cuyunii or G. stergiosi. Moreover, G. dacrya has a shallower body than G. cuyunii and a smaller preorbital region, and longer pelvic fin than G. stergiosi. Guianacara dacrya has a broader lower pharyngeal jaw (length $66 \%$ of width) than either G. stergiosi $(82 \%)$ or $G$. cuyunii $(79 \%)$, and also a wing-like expansion of the lateral border surrounding the posterior half of the dentigerous area we could not observe in clear and stained specimens of $G$. owroewefi (ANSP 187132), G. stergiosi, and G. cuyunii (see López-Fernández et al., 2006) or in figures or descriptions of tooth plates from other species of Guianacara (Kullander \& Nijssen, 1989). Guianacara dacrya differs from other Guianacara in the possession of a modified infraorbital stripe in adult specimens (faded across the cheek); in at least $G$. owroewefi, if the infraorbital stripe fades (probably as an ontogenetic color change sometimes exacerbated by preservation) it does so primarily along the ventral-most region of the stripe, on the interopercle and preopercle, opposite to that observed in G. dacrya, which was always dark on the preopercle and interopercle.

Guianacara dacrya has been collected from a broad region of southern and central Guyana that encompasses both the Essequibo and Branco River drainages. It is not clear how far G. dacrya reaches into the rio Branco basin, but the presence of Guianacara in the rio Uraricoera (see Comparative material) may suggest that this species could range at least as far as northern Brazil. However, the three specimens of Guianacara from northern Brazil could not be positively identified as $G$. dacrya due to their small size and poor state of preservation. 
The lack of any apparent morphological differentiation in specimens from the Essequibo and the Branco River drainages (Fig. 6) suggests that G. dacrya is capable of moving between the basins, although genetic evidence is not available. Movement most likely occurs in the wet-season, when large expanses of the Rupununi savannas between the Kanuku and Pakaraima mountains are flooded. At least in some years the annual flood cycle allows full connection between both basins (Lowe-McConnell, 1964). Several other fish species have been identified with distributions in both drainages (e.g., Rhinodoras armbrusteri [Doradidae, Sabaj et al., 2008], Cichla ocellaris [HLF et al., pers. obs. ROM 85713, ROM 86140]), but other species are known to have distributions restricted to one of the drainages (e.g., Cichla temensis in the rio Branco, HLF pers. obs. ROM 86127 and see Willis et al., 2010). To our knowledge, only one genetic study has shown a significant role for the Rupununi savannas as a corridor between the Amazon and Essequibo basins (Lovejoy $\&$ de Araújo, 2000), but it is likely that the rainy season connection between the two basins affects different taxa in different ways, and that ecological differences between basins play a major role in determining which species are able to cross the divide, as is the case in the connection between the Amazon and Orinoco basins through the Casiquiare River (Winemiller et al., 2008, Willis et al., 2010).

While G. cuyunii is also found in the Essequibo drainage (upper Cuyuni River in Venezuela), G. dacrya and G. cuyunii do not appear to be sympatric in rivers of the Essequibo basin. Essentially the distribution of both species seems to be limited to the upper and/or middle reaches of the Cuyuni and Essequibo with the genus being absent in the lower portion of the Essequibo basin where the two rivers intersect. This distributional pattern is similar to previously described Guianacara species which are predominantly allopatric, with the exception of G. oelemariensis and G. owroewefi which are sympatric within the Oelemari River in Suriname (Kullander \& Nijssen, 1989). This pattern of allopatry suggests a strong association with habitat characteristics (e.g., clear water, rocky structure, low conductivity) that are generally encountered in rivers originating in the Guiana shield highlands and are absent in floodplains of the lower Essequibo. Thus the distribution of all species of Guianacara appears to be tightly linked to ecological requirements.

Comparative material. Guianacara sp. aff. dacrya: INPA 1404, 1, $45.8 \mathrm{~mm}$ SL, Brazil, Roraima, rio Uraricoera, Branco drainage, Maraca Island at Paraná do Firmino, 18 Sep 1987, Ferreira et al.; INPA 7708, 2, 25.2-35.6 mm SL, rio Uraricoera, Branco drainage, Maraca Island at rapids downstream from Uraricoera Island, 13 Mar 1988, M. Jégu. Guianacara owroewefi: ANSP 187132, 25, 19.4-76.6 mm SL, Suriname, Sipalawini, Lawa River, Marowijne drainage, $8 \mathrm{~km} \mathrm{SSW}$ of Anapaike/Kawemhakan Airstrip, 03¹9'31'N 5403'48'W, 18 Apr 2007, J. Lundberg et al. Guianacara stergiosi: ROM 82271, 5, 40.7-63.5 mm SL, Venezuela, Bolivar, río Aro, 5 Feb 2004, Willis et al.; ROM 84606, 5, 24.8-41.5 mm SL, Venezuela, Bolivar, río Claro, at bridge on road to Guri, $07^{\circ} 54^{\prime} 55.4^{\prime \prime} \mathrm{N}$ 6305'26.4’'W, 25 Jan 2000, H. López-Fernández et al.

\section{Acknowledgements}

We are grateful to C. Bernard, D. C. Taphorn, K. O. Winemiller, C. Montaña, G. Ortí, D. Bloom, M. Tobler, R. King, and K. Sugrim for their help in the field and to M. Sabaj-Pérez (ANSP), J. Armbruster and D. Werneke (AUM), L. Rapp PyDaniel and M. Rocha (INPA) for the loan of specimens in their care. Thanks to E. Holm (ROM) for his assistance with specimens and field data. A. Guidotti (ROM) helped producing Fig. 4. Donald C. Taphorn read an earlier version of the manuscript and offered important suggestions for its improvement. This project was funded by research grants from the ROM Governors and an NSERC Discovery Grant to HLF, and by an Alexander Graham Bell Canada Graduate Scholarship from NSERC to JHA. This paper is part of an ongoing collaboration between the Center for the Study of Biodiversity, University of Guyana and the Royal Ontario Museum to document the fish diversity of Guyana.

\section{Literature Cited}

Bookstein, F. L. 1991. Morphometric tools for landmark data: Geometry and biology. Cambridge University Press, Cambridge.

Bookstein, F. L. 1996. Landmark methods for forms without landmarks: morphometrics of group differences in outline shape. Medical Image Analysis, 1(3): 225-243.

Farias, I. P., G. Ortí, I. Sampaio, H. Schneider \& A. Meyer. 1999. Mitochondrial DNA phylogeny of the family Cichlidae: monophyly and fast molecular evolution of the Neotropical assemblage. Journal of Molecular Evolution, 48: 703-711.

Jacques, P. 1902. Cichlidé nouveau de la Guyane française. Bulletin du Musée National d'Histoire Naturelle, 8: 417-418.

Kullander, S. O. 1986. Cichlid Fishes of the Amazon River Drainage of Peru. Swedish Museum of Natural History, Stockholm.

Kullander, S. O. 1996. Heroina isonycterina, a new genus and species of cichlid fish from Western Amazonia, with comments on cichlasomine systematics. Ichthyological Exploration of Freshwaters, 7: 149-172.

Kullander, S. O. 2003. Cichlidae (Cichlids). Pp. 605-654. In: Reis, R. E., S. O. Kullander \& C. J. Ferraris Jr. (Eds.) Checklist of the Freshwater Fishes of South and Central America. Porto Alegre, Edipucrs.

Kullander, S. O. \& H. Nijssen. 1989. The cichlids of Surinam. E.J. Brill, Leiden.

Leviton, A. E., R. H. Gribbs Jr., E. Heal \& C. E. Dawson. 1985. Standards in Herpetology and Ichthyology: part I. Standard symbolic codes for institutional resource collections in herpetology and ichthyology. Copeia, 1985: 802-832.

López-Fernández, H., D. C. Taphorn \& S. O. Kullander. 2006. Two new species of Guianacara from the Guiana Shield of Eastern Venezuela (Perciformes: Cichlidae). Copeia, 2006(3): 384-395.

López-Fernández, H. \& K. O. Winemiller. 2010. Multilocus phylogeny and rapid radiation in Neotropical cichlid fishes (Perciformes: Cichlidae: Cichlinae). Molecular Phylogenetics and Evolution, 55: 1070-1086.

Lovejoy, N. R. \& M. L. G. de Araújo. 2000. Molecular systematics, biogeography and population structure of Neotropical freshwater needlefishes of the genus Potamorrhaphis. Molecular Ecology, 9: 259-268. 
Lowe-McConnell, R. H. 1964. The fishes of the Rupununi savanna district of British Guiana, South America. Part 1. Ecological groupings of fish species and effects of the seasonal cycle on the fish. Zoological Journal of the Linnean Society, 45: 103144.

Lowe-McConnell, R. H. 1969. The cichlid fishes of Guyana, South America, with notes on their ecology and breeding behaviour. Zoological Journal of the Linnean Society, 48:255-302.

Reist J. D. 1986. An empirical evaluation of several univariate methods that adjust for size variation in morphometric data. Canadian Journal of Zoology, 63: 1429-1439.

Rohlf, F. J. 2002. Geometric morphometrics in phylogeny. In: Morphology, Shape and Phylogeny. Systematics Association Special Volume Series, 64: 175-193.

Rohlf, F. J. 2003. tpsRelw, relative warps analysis, version 1.36. Department of Ecology and Evolution, State University of New York at Stony Brook.

Rohlf, F. J. \& D. Slice. 1990. Extensions of the Procrustes method for the optimal superimposition of landmarks. Systematic Zoology, 39: 40-59.

Sabaj-Pérez, M. H. 2010. Standard symbolic codes for institutional resource collections in herpetology and ichthyology: an Online Reference. Verson 1.5 (4 Oct 2010). Electronically accessible at http://www.asih.org/, American Society of Ichthyologists and Herpetologists, Washington, DC.

Sabaj, M. H., D. C. Taphorn \& O. E. Castillo. 2008. Two new species of thicklip thornycats, genus Rhinodoras (Teleostei: Siluriformes: Doradidae). Copeia, 2008(1): 209-226.

Taylor, W. R., \& G. C. van Dyke. 1985. Revised procedures for staining and clearing small fishes and other vertebrates for bone and cartilage study. Cybium, 9: 107-119.

Willis, S. C., M. Nunes, C. G. Montaña, I. P. Farias \& N. R. Lovejoy. 2010. The Casiquiare river acts as a corridor between the Amazonas and Orinoco river basins: biogeographic analysis of the genus Cichla. Molecular Ecology, 19: 1014-1030.

Winemiller, K. O., H. López-Fernández, D. C. Taphorn, L. G. Nico \& A. B. Duque. 2008. Fish assemblages of the Casiquiare river, a corridor and zoogeographical filter for dispersal between the Orinoco and Amazon basins. Journal of Biogeography, 35: 15511563.

Accepted November 2, 2010

Published March 31, 2011 